\title{
Motivación autodeterminada en adultos mayores practicantes de ejercicio físico
}

\section{Self-determination motivation in elderly practitioners of physical exercise}

\section{Motivación autodeterminada en adultos mayores practicantes de ejercicio físico}

\author{
Pablo Jorge Marcos Pardo ${ }^{1}$, Francisco Javier Orquín Castrillón ${ }^{1}$, Noelia Belando Pedreño y Juan Antonio Moreno-Murcia ${ }^{2}$ \\ 1 Grupo de Investigación en Salud, Actividad Física, Fitness y Comportamiento Motor, Universidad Católica de Murcia (España), \\ 2 Departamento de Psicología de Salud, Universidad Miguel Hernández de Elche (España)
}

\begin{abstract}
Resumen: El objetivo de este estudio fue comprobar la capacidad predictiva de la valoración del comportamiento autónomo, las necesidades psicológicas básicas y el índice de autodeterminación sobre los motivos de práctica autodeterminados (fitness/salud, social y disfrute) en adultos mayores. La muestra estuvo integrada por 237 practicantes, con edades comprendidas entre 65 y 85 años $(M=74.51, D T=8.04)$. El análisis de regresión lineal múltiple mostró que el motivo fitness/salud fue predicho positivamente por la competencia percibida y el índice de autodeterminación. A su vez, los motivos social y disfrute fueron predichos por la valoración del comportamiento autónomo, la competencia percibida y la relación con los demás. Estos resultados destacan la importante contribución que los factores psicosociales podrían tener en la práctica de ejercicio físico saludable para el envejecimiento activo después de la edad adulta.

Palabras clave: motivación, necesidades psicológicas básicas, ejercicio físico, adultos mayores.

Abstract: The aim of this study was to test the predictive ability of the autonomous behavioral assessment, basic psychological needs and the rate of self-determination on the grounds of self-determined practice (fitness / health, social and enjoy) in elderly. The sample consisted of 237 trainees, aged between 65 and 85 years $(M=74.51, S D=8.04)$. The multiple linear regression analysis showed that the motive fitness/health was positively pre-
\end{abstract}

dicted by perceived competence and self-determination index. In turn, social and enjoy the grounds were predicted by the assessment of autonomous behavior, perceived competence and the relationship with others. These results show the relevance of psychosocial factors in physical exercise healthy active aging in the population.

Keywords: motivation, basic psychological needs, physical exercise, old adult. Resumo: El objetivo de este estudio fue comprobar la capacidad predictiva de la valoración del comportamiento autónomo, las necesidades psicológicas básicas y el índice de autodeterminación sobre los motivos de práctica autodeterminados (fitness/salud, social y disfrute) en adultos mayores. La muestra estuvo integrada por 237 practicantes, con edades comprendidas entre 65 y 85 años $(\mathrm{M}=74.51$, DT $=8.04)$. El análisis de regresión lineal múltiple mostró que el motivo fitness/salud fue predicho positivamente por la competencia percibida y el índice de autodeterminación. A su vez, los motivos social y disfrute fueron predichos por la valoración del comportamiento autónomo, la competencia percibida y la relación con los demás. Estos resultados destacan la importante contribución que los factores psicosociales podrían tener en la práctica de ejercicio físico saludable para el envejecimiento activo después de la edad adulta.

Palabras Clave: motivación, necesidades psicológicas básicas, ejercicio físico, adultos mayores.

\section{Introducción}

Un creciente cuerpo de investigación considera la motivación autodeterminada como varible predictora de consecuencias positivas a nivel psicológico, social y afectivo en adultos mayores practicantes de ejercicio físico (Bartolomé, Rebollo, Pérez-Aranda y Paramio, 2013; Ferrand, Nasarre, Hautier, y Bonnefoy, 2012). Siguiendo las recomendaciones de Krech (2011), el envejecimiento activo se relaciona con ser activo social y mentalmente a través de actividades recreativas, de carácter voluntario o remuneradas, actividades culturales, sociales y actividades educativas (Ostir, Cohen Mansfield, Levielle, Volpato, y Guralnik, 2003). Asimismo, una reducida funcionalidad física en esta población se asocia con

Dirección para correspondencia [Correspodence address]: Pablo Jorge Marcos Pardo, Facultad de Ciencias de la Actividad Física y del Deporte (Universidad Católica de Murcia), Campus de los Jerónimos, s/n. 30107. Guadalupe, Murcia (Espańa). E-mail: pmarcos@ucam.edu resultados negativos en la vida posterior, incluyendo mayor riesgo de demencia y mortalidad (Hamer, Batty, Kivimaki, y Stamatakis, 2011; Zimmer, Martin, Jones, y Nagin, 2014). En este sentido, resulta necesario profundizar en los factores psico-sociales que podrían contribuir en el grado de motivación más o menos autodeterminada de los adultos mayores hacia la práctica de ejercicio físico.

Para el análisis de este propósito una de las teorías más prolíferas que se ha encargado de estudiar la motivación humana es la teoría de la autodeterminación (Deci y Ryan, 1985, 2000, 2012). El nivel de motivación más o menos autodeterminado del practicante va a estar influenciado en gran medida por la interacción de éste con el técnico deportivo, el grupo de iguales y demás agentes sociales (Deci y Ryan, 1991). Un contexto de ejercicio físico en el que se valore el comportamiento autónomo de los practicantes, podría ga- 
rantizar la satisfacción de las necesidades psicológicas básicas (autonomía, competencia y relación con los demás). Dichas necesidades median la relación entre los factores ambientales y el grado de motivación experimentada.

En esta línea, estudios realizados por Conroy y Coastsworth (2007) en practicantes jóvenes, muestran que la valoración de los comportamientos autónomos, entendido como la capacidad de empatía que tiene el técnico deportivo con los practicantes, podría ser determinante para detectar sus necesidades, sus sentimientos, ofrecerles la información apropiada, significativa y dar oportunidad de elección en las tareas (Ryan y Deci, 2000). Parece que, cuando los practicantes perciben que el técnico deportivo se comporta de forma empática y se sienten comprendidos y apoyados por él o por ella, se ven favorecidas sus relaciones sociales (Balaguer, Castillo, y Duda, 2008). De manera que, la valoración del comportamiento autónomo mostrado por los profesionales deportivos, podría influir en que los adultos mayores se sintieran autónomas, competentes, involucradas en la toma de decisiones, responsables y que percibieran una relación positiva con el entorno social, experimentando mayor sentimiento de satisfacción con la práctica. En concordancia, Ryan, Frederick, Lepes, Rubio, y Sheldon (1997) reflejan cinco dimensiones fundamentales que podrían llevar a una persona a realizar ejercicio físico. Nos muestran el disfrute como la prioridad al factor diversión en la práctica, la apariencia (la importancia de la imagen), el fitness/salud (mejorar aptitudes fisiológicas), la competencia (afrontar desafíos) y el motivo de practica social (conocer personas y hacer amistades). En el presente estudio se han analizado los motivos salud, social y disfrute como intenciones más autodeterminadas (intrínsecas a la persona) para realizar ejercicio físico. Al respecto, varias investigaciones (Hellín, Moreno, y Rodríguez, 2004; Moreno, Cervelló, y Martínez, 2007; Martínez del Castillo, García, Graupera, Jiménez-Beatty, Martín, y Pouso, 2002) observaron que la salud, además de la diversión y la posibilidad de relacionarse con los demás son los motivos más aludidos que les llevan a las personas mayores a practicar actividad física.

Según lo expuesto y atendiendo a los estudios revidados, profundizar en el conocimiento de los motivos más autodeterminados de práctica de ejercicio físico de los adultos mayores podría tener consecuencias positivas para el bienestar general en esta población. Por lo que se propuso comprobar la capacidad predictiva del comportamiento autónomo, las necesidades psicológicas básicas y el índice de autodeterminación sobre los motivos de práctica fitness/salud, social y disfrute en los adultos mayores. Asimismo, hipotetizamos que la valoración del comportamiento autónomo, la autonomía, competencia percibida y la relación con los demás predirán los motivos de práctica más autodeterminados (salud, social y disfrute) en los adultos mayores.

\section{Método}

\section{Participantes}

La muestra del estudio estuvo formada por 237 practicantes (94 hombres y 143 mujeres) con edades comprendidas entre los 65 y 85 años $(M=74.51 ; D T=8.04)$, pertenecientes a una población de practicantes de diversos centros deportivos. Se seleccionaron aleatoriamente cuatro centros de de la Region de Murcia con un reparto equitativo en el número de mujeres y hombres. Del total de la muestra El $77.4 \%$ practicaba ejercicio físico 2-3 días por semana y el 22.6\% lo realizaban más de 3 días semanales.

\section{Medidas}

Valoración del comportamiento autónomo. Se empleó la dimensión valoración del comportamiento autónomo de Conde, Sáenz-López, Carmona, González-Cutre, Martínez-Galindo, y Moreno, (2010) del Autonomy-Supportive Coaching Questionnaire de Conroy y Coastsworth (2007), adaptado al contexto del ejercicio físico saludable. Esta dimensión se evalúa a través de cuatro ítems (e.g. "Mi técnico me valora por las cosas que elijo hacer en la práctica"). El cuestionario estaba encabezado por la frase "En mis prácticas de ejercicio físico...", respondiéndose mediante una escala de respuesta tipo Likert de 1 (Totalmente en desacuerdo) a 7 (Totalmente de acuerdo). La consistencia interna fue de .79.

Necesidades psicológicas básicas en el ejercicio. Se utilizó la versión en español (Sánchez y Núñez, 2007) de la Escala de las Necesidades Psicológicas Básicas en el Ejercicio (Vlachopoulos y Michailidou, 2006). El inventario constaba de 12 ítems agrupados en tres dimensiones (cuatro ítems por dimensión): autonomía (e.g. "El programa de ejercicio físico que sigo se ajusta a mis intereses"), competencia (e.g. "He tenido una gran progresión con respecto al resultado perseguido") y relación con los demás (e.g. "Me siento muy cómodo/a cuando hago ejercicio con los/as demás practicantes"). La frase previa fue "En mis prácticas de ejercicio físico..." y las respuestas fueron puntuadas con una escala tipo Likert que oscila entre 1 (Totalmente en desacuerdo) y 5 (Totalmente de acuerdo). Se obtuvieron valores alfa de Cronbach de .71 para autonomía, .72 para competencia y .91 para relación con los demás.

Motivación. Para medir la motivación hacia la actividad física en el contexto del ejercicio físico se utilizó la BREQ-3 (González-Cutre, Sicilia, y Fernández, 2010). El instrumento está compuesto por 23 ítems: cuatro para la regulación intrínseca (e.g. "Porque disfruto con las sesiones de ejercicio físico"), cuatro para la regulación integrada (e.g. "Porque está de acuerdo con mi forma de vida"), tres para la regulación identificada (e.g. "Porque para mí es importante hacer ejer- 
cicio físico regularmente"), cuatro para la regulación introyectada (e.g. "Porque siento que he fallado cuando no he realizado un rato de ejercicio físico"), cuatro para la regulación externa (e.g. "Porque mis amigos/familia/pareja me dicen que debo hacerlo") y cuatro para la desmotivación (e.g. "Pienso que hacer ejercicio físico es una pérdida de tiempo"). Las respuestas encabezadas por el enunciado "Yo hago ejercicio físico..." se respondían mediante una escala tipo Likert de 0 (Nada verdadero) a 4 (Totalmente verdadero). La consistencia interna fue de .70 para la regulación intrínseca, .81 para la regulación integrada, .67 para la regulación identificada, .62 para la regulación introyectada, .80 para la regulación extrínseca y .60 para la desmotivación. Dado el reducido número de ítems que componen los factores regulación identificada, regulación introyectada y la desmotivación, la consistencia interna obtenida se consideró aceptable (Hair, Anderson, Tatham, y Black, 1998). Con los factores de esta escala se calculó el índice de autodeterminación (IAD), que indicaba cómo de autodeterminada era la motivación de la población mayor. Para calcular el índice de autodeterminación (IAD) (Vallerand, 1997) se utilizó la siguiente fórmula: [(2 x Regulación intrínseca) + Regulación identificada + Regulación integrada $]-[$ (Regulación externa + Regulación introyectada) / 2] - (2x Desmotivación).

Motivos de práctica para el ejercicio físico. De la versión al castellano (Moreno, Cervello y Martinez, 2007) de Motives for Physical Activity Measure-Revised de Ryan, Hicks, y Midgley (1997), se utilizaron las dimensiones fitness/salud, social y disfrute. El factor fítness/salud está formado por seis ítems (e.g. "Porque quiero estar en buena forma física"), la dimensión social está compuesta por cinco ítems (e.g. "Porque me gusta estar con mis amigos”), mientras que la dimensión disfrute está formada por siete ítems (e.g. "Porque es divertido"). La sentencia previa fue "Realizo actividad físico-deportiva...". Las respuestas se establecieron con una escala tipo Likert que oscilaba entre 1 (Totalmente en desacuerdo) y 7 (Totalmente de acuerdo). La consistencia internada fue de .85 para el motivo fitness/salud, .86 para el motivo social y .83 para el motivo disfrute.

\section{Procedimiento}

\section{Procedimiento}

Los cuestionarios se administraron a una población de adultos mayores practicantes de ejercicio físico. Antes de iniciar el estudio, se presentó la propuesta de investigación a los responsables de los centros deportivos y se les informó del objetivo de la investigación con el propósito de contar con su autorización y conformidad. Para la administración de los cuestionarios se procedió a informar a los participantes integrantes de la muestra de estudio, acerca del objetivo del mismo. Para la correcta cumplimentación de los cuestionarios de forma escrita fue necesaria la ayuda del investigador en personas con problemas de visión y comprensión lectora. Se explicó el contenido de algunos ítems sin influir en sus decisiones y se garantizó la confidencialidad de sus respuestas y de la participación voluntaria. El tiempo requerido para cumplimentar el cuestionario fue de 25 minutos.

\section{Análisis de datos}

Para comprobar la relación e importancia de las distintas variables se calcularon los estadísticos descriptivos y correlaciones de todas las variables. Para el cálculo de la consistencia interna se utilizó el alfa de Cronbach. Para comprobar la predicción de la valoración del comportamiento autónomo, las necesidades psicológicas básicas y el índice de autodeterminación (como variables independientes) sobre los motivos fitness/salud, social y disfrute (variables dependientes), se realizó un análisis de regresión múltiple. Los datos fueron analizados con el programa estadístico SPSS 20.0.

\section{Resultados}

\section{Análisis descriptivo y de correlaciones bivariadas}

La valoración del comportamiento autónomo presentó una media de 4.68. El mediador psicológico más valorado fue el de relación con los demás, seguido de la autonomía y de la competencia percibida. El índice de autodeterminación osciló de -10.17 a 13.92, con un valor medio de 9. El motivo de práctica deportiva fitness/salud obtuvo mayor puntuación que el motivo disfrute y el motivo social. En el análisis de correlación se observó que la variable valoración del comportamiento autónomo correlacionó positivamente con la autonomía y con la relación con los demás. Las tres necesidades psicológicas básicas correlacionaron positivamente entre sí, con el índice de autodeterminación y con los motivos de práctica autodeterminados. Por su parte, el índice de autodeterminación y los tres motivos de práctica correlacionaron de forma positiva con todas las variables excepto con la valoración del comportamiento autónomo (tabla 1). 
Tabla 1. Estadísticos Descriptivos y Correlaciones de Todas las Variables.

\begin{tabular}{llllllllllll}
\hline Variables & $M$ & $D T$ & $\mathrm{R}$ & 1 & 2 & 3 & 4 & 5 & 6 & 7 & 8 \\
\hline 1. Valoración del comportamiento autónomo & 4.68 & 1.54 & $1-7$ & - & $.73^{* *}$ & .08 & $.23^{* *}$ & .03 & .05 & .16 & .13 \\
2. Autonomía & 4.61 & .57 & $1-5$ & - & - & $.67^{* *}$ & $.54^{* *}$ & $.31^{* *}$ & $.50^{* *}$ & $.47^{* *}$ & $.44^{* *}$ \\
3. Competencia & 4.57 & .51 & $1-5$ & - & - & - & $.66^{* *}$ & $.44^{* *}$ & $.62^{* *}$ & $.61^{* *}$ & $.63^{* *}$ \\
4. Relación con los demás & 4.79 & .47 & $1-5$ & - & - & - & - & $.46^{* *}$ & $.47^{* *}$ & $.73^{* *}$ & $.73^{* *}$ \\
5. IAD & 9.00 & 1.90 & - & - & - & - & - & - & $.44^{* *}$ & $.41^{* *}$ & $.41^{* *}$ \\
6. Fitness/salud & 6.65 & .63 & $1-7$ & - & - & - & - & - & - & $.59^{* *}$ & $.67^{* *}$ \\
7. Social & 6.15 & 1.19 & $1-7$ & - & - & - & - & - & - & - & $.87^{* *}$ \\
8. Disfrute & 6.36 & .80 & $1-7$ & - & - & - & - & - & - & - & - \\
\hline
\end{tabular}

Nota: ${ }^{*} p<.05 ;{ }^{* *} p<.01 ; M=$ media; $D T=$ desviación típica; $\mathrm{R}=$ rango.

Tabla 2. Resumen del Análisis de Regresión Múltiple de los Motivos de Práctica Fitness/Salud, Social y Disfrute Según la Valoración del Comportamiento Autónomo, Mediadores Psicológicos e Índice de Autodeterminación

\begin{tabular}{lcccc}
\hline & $B$ & $E T B$ & $b$ & $\Delta R 2$ \\
\hline Fitness/Salud & & & & $.44^{* *}$ \\
Valoración & .00 & .03 & .00 & \\
Autonomía & .16 & .10 & .15 & \\
Competencia & .52 & .13 & $.42^{* *}$ & \\
Relación con los demás & .03 & .13 & .02 & \\
IAD & .07 & .03 & $.20^{*}$ & \\
Social & & & & $.55^{* *}$ \\
Valoración & .20 & .06 & $.26^{*}$ & \\
Autonomía & -.01 & .17 & -.00 & \\
Competencia & .49 & .21 & $.21^{*}$ & \\
Relación con los demás & 1.44 & .21 & $.57^{* *}$ & \\
IAD & .03 & .04 & .05 & \\
Disfrute & & & & $.57^{* *}$ \\
Valoración & .43 & .19 & $.21^{*}$ & \\
Autonomía & -1.18 & .11 & -.08 & \\
Competencia & .48 & .14 & $.31^{* *}$ & \\
Relación con los demás & .94 & .14 & $.56^{* *}$ & \\
IAD & .02 & .03 & .05 & \\
\hline
\end{tabular}

Nota: ${ }^{*} p<.05 ;{ }^{* *} p<.01 ; B=$ coeficiente de regresión parcial no estandarizado; $E T=$ Error típico; $b=$ coeficiente de regresión parcial estandarizado; $R 2=$ coeficiente de determinación.

\section{Análisis de regresión lineal múltiple}

Se llevaron a cabo tres análisis de regresión lineal por pasos en cada variable dependiente (fitness/salud, social y disfrute). Para incluir dichas variables comprobamos que no eran colineales mediante los estadísticos de colinealidad (tolerancia y
FIV). En cada uno de ellos se introdujo en el primer paso la variable valoración, en el segundo paso los mediadores psicológicos y en el tercer paso la motivación autodeterminada. El resultado del análisis de regresión lineal múltiple final para el motivo fitness/salud explicó un 44\% de varianza total explicada, siendo predictores positivos la competencia percibida y el índice de autodeterminación. Para los motivos social y disfrute la varianza explicada fue de $55 \%$ y $57 \%$, respectivamente, donde la valoración del comportamiento autónomo, la competencia percibida y la relación con los demás fueron predictores positivos (tabla 2).

\section{Discusión}

Son evidentes los efectos positivos de la actividad física sobre el bienestar físico, psicológico y de satisfacción con la vida de los adultos mayores (Barriopedro, Erańa, y Mallol, 2001; Faulkner y Biddle, 2002; De García, Marín, y Bohórquez, 2012). Para abordar esta cuestión, es importante conocer los factores psicosociales que podrían influir en los motivos de práctica más autodeterminados de esta población. Por ello, el estudio tuvo como objetivo comprobar la capacidad predictiva de la valoración del comportamiento autónomo, las necesidades psicológicas básicas y el índice de autodeterminación sobre los motivos de práctica fitness/ salud, social y disfrute. Al considerar todos los motivos conjuntamente no se confirma la hipótesis inicial. El motivo fitness/salud fue predicho positivamente por la competencia percibida y el índice de autodeterminación, correlacionando positivamente entre sí. En este sentido, estudios anteriores (De García y Marcó, 2000; Ostir et al., 2003; Piedras-Jorge, Meléndez-Moral, y Tomás-Miguel, 2010) mostraron que la práctica de ejercicio físico no solo influye en la mejora de la salud física sino que tiene implicaciones positivas en el estado cognitivo y en el bienestar psicológico de los adultos mayores. Así que, una persona que se percibe competente en la práctica podría tener una actitud más 
positiva y encontrarse motivada (Pieron, 1998). En esta línea, en el estudio de André y Dishman (2012), las personas con mayor motivación autodeterminada tendían a percibir más ventajas del ejercicio físico y una mejor calidad de vida. Además, una mayor motivación autodeterminada favorece el nivel de práctica y la adherencia al ejercicio físico en personas mayores (André y Dishman, 2012; Dacey, Baltzell, y Zaichkowsky, 2008).

Por otro lado, los motivos de práctica social y disfrute fueron predichos positivamente por la valoración del comportamiento autónomo, la competencia percibida y la relación con los demás, aunque se relacionaron positivamente con las tres necesidades psicológicas básicas (autonomía, competencia y relación con los demás) y con el índice de autodeterminación. En concordancia con estos resultados, la teoría de la autodeterminación afirma que el apoyo a la autonomía es el elemento esencial para la satisfacción de las necesidades psicológicas (Deci y Ryan, 1987). De manera que, si son satisfechas las necesidades psicológicas básicas que median el contexto social, podría aumentar la motivación intrínseca, y ésta a su vez, puede presentar consecuencias positivas sobre el bienestar y el desarrollo personal (Standage y Treasure, 2002).

En analogía con los resultados observados, Moreno-Murcia, Águila, y Borges (2011) en un estudio realizado con población adulta, encontraron que la competencia percibida, la relación con los demás y la motivación intrínseca predijeron positivamente los motivos sociales. Parece que los efectos positivos de la actividad física podrían deberse a la correcta combinación en el contexto social del ejercicio, a su significación personal y al entorno físico en el que se desarrolla (Roth, Bachtler, y Fillingim, 1990). Resulta notable la importancia del apoyo social (Deci y Ryan, 2008; Fernández y Manrique-Abril, 2010; Graupera, Martínez del Castillo, y Martín, 2003) en la realización de actividad física entre adultos mayores para conseguir en ellos conductas más autodeterminadas pudiendo mejorar su autoestima, su salud y en definitiva su calidad de vida.

Contribuyendo a la literatura revisada, no obstante, el presente estudio tiene varias limitaciones que deben tenerse en cuenta en futuras investigaciones. En primer lugar, el pequeño tamaño de la muestra nos impide usar los análisis más detallados. En particular, la necesidad de autonomía no predice los motivos de práctica objeto de estudio. Nos encontramos ante una limitación del estudio que podría explicarse por la falta de concreción del concepto de autonomía en el contexto español. La población de mayor edad parece concebir el contexto social como oportunidad de un mayor apoyo psico-social, percepción de afecto (empatía) y relación con los demás, más que la importancia de la cesión de autonomía a través de la toma de decisiones sobre la actividad a realizar. Sería necesario revisar la redacción del concepto de autonomía percibida en el contexto español y esta población en concreto.

En este intento de profundizar en el conocimiento de los factores ambientales y psicológicos que podrían determinar las intenciones de práctica deportiva de esta población, son pertinentes futuros estudios experimentales de desarrollo longitudinal. Diversas líneas de investigación enfocadas a analizar las consecuencias positivas de la adaptación de los programas de ejercicio físico a las características funcionales y psico-sociales de las personas mayores de manera que sientan satisfechos sus objetivos personales. De modo que, esta población encuentre en el ejercicio físico una herramienta útil para mejorar su calidad de vida y su autoconfianza, así como contribuir a la consecución de objetivos o logros que los motive a continuar (Hardclastle y Taylor, 2001; Van Norman, 1998). Asimismo, serían interesante llevar a cabo estudios que analicen la importancia del grupo de los iguales en la persistencia en la práctica de actividad física en esta etapa vital.

Por último, destacar que debido a la posible influencia del distinto rango de respuesta de los instrumentos en el análisis estadístico sería necesario en el futuro comprobar el efecto del rango en las respuestas y lógicamente en las consecuencias obtenidas.

En conclusión, el presente estudio podría servir a los técnicos deportivos para que conozcan la importancia de valorar la actitud de los practicantes y saber de la importancia de utilizar estrategias motivacionales que garanticen la satisfacción de las necesidades psicológicas básicas. Promoviendo en los adultos mayores motivos más autodeterminados de práctica deportiva y consecuencias positivas para su salud general como la adherencia a la práctica.

\section{Aplicaciones prácticas}

Los resultados obtenidos en coherencia con los estudios considerados, podrían contribuir como guía para la planificación de programas de ejercicio físico en adultos mayores, bajo metodologías que fomenten la autonomía de los practicantes (tareas abiertas, fomento de la iniciativa, elección de actividades, etc.) ya que parecen relacionarse con una consecución de las necesidades psicológicas básicas (Álvarez et al., 2009) así como una mayor motivación intrínseca (Moreno et al., 2009; Balaguer, Castillo, y Duda 2008). En este sentido, nos unimos a algunas propuestas (Marcos, 2010; Moreno-Murcia y Marcos, 2010; Moreno, Cervelló, González-Cutre, y Del Villar, 2011; Moreno-Murcia, Huéscar, y Hellín, 2014), en las que se establecen una serie de estrategias motivacionales para conseguir unas conductas más adaptativas al ejercicio físico y promover una serie de actividades para favorecer la autonomía, la satisfacción de las necesidades psicológicas básicas y la motivación intrínseca. 
Agradecimientos.- Esta investigación fue realizada por el Grupo de Investigación en Salud, Actividad Física, Fitness y Comportamiento Motor (GISAFFCOM), gracias al pro- yecto PMAFI/03/12 avalado por la Universidad Católica de Murcia (España).

\section{Referencias}

1. Álvarez, M. S., Balaguer, I., Castillo, I., y Duda, J. L. (2009).Coach autonomy support and quality of sport engagement in young soccer players. The Spanish Journal of Psychology, 12(1), 138-148.

2. André, N., y Dishman, R. K. (2012). Evidence for the construct validity of self-motivation as a correlate of exercise adherence in French older adults. Journal of Aging and Physical Activity, 20, 231-245.

3. Balaguer, I., Castillo, I., y Duda, J. (2008). Apoyo a la autonomía, satisfacción de las necesidades, motivación y bienestar en deportistas de competición: un análisis de la teoría de la autodeterminación. Revista de Psicología del Deporte, 17(1), 123-139.

4. Barriopedro, M. I., Erańa, I., y Mallol, Ll. (2001). Relación de la actividad física con la depresión y la satisfacción con la vida en la tercera edad. Revista de Psicología del Deporte, 10(2), 239-246.

5. Conde, C., Sáenz-López, P., Carmona, J., González-Cutre, D., Martínez-Galindo, C., y Moreno, J. A. (2010). Validación del Cuestionario de Percepción de Soporte de la Autonomía en el Proceso de Entrenamiento (ASCQ) en jóvenes deportistas españoles. Estudios de Psicología, 31, 145-157. doi: 10.1174/021093910790744527

6. Conroy, D., y Coatsworth, J. (2007). Assessing autonomy-supportive coaching strategies in youth sport. Psychology of Sport and Exercise, 8, 671-684. doi: 10.1016/j.psychsport.2006.12.001

7. Dacey, M., Baltzell, A., y Zaichkowsky, L. (2008). Older adults' intrinsic and extrinsic motivation toward phisical activity. American Journal of Health Behavior, 32(6), 570-582. doi: 10.5993/AJHB.32.6.2

8. De García, A. J., Marín, M., y Bohórquez, M. (2012). Autoestima como variable psicosocial predictora de la actividad física en personas mayores. Revista de Psicología del Deporte, 21(1), 195-200.

9. De Garcia, M., y Marcó, M. (2000). Efectos psicológicos de la actividad física en personas mayores. Psicothema, 12(2), 285-29.

10. Deci, E. L., y Ryan, R. M. (1985). Intrinsic motivation and self-determination in human behavior. New York: Plenum Press.

11. Deci, E. L., y Ryan, R. M. (1987). The support of autonomy and the control of behavior. Journal of Personality and Social Psychology, 53, 1024-1037.

12. Deci, E. L., y Ryan, R. M. (1991). A motivational approach to self: Integration in personality. En R. Dienstibier (Ed.), Nebraska symposium on motivation: Vol. 38. Perspectives on motivation (pp. 237-288). Lincoln, NE: University of Nebraska Press.

13. Deci, E. L., y Ryan, R. M. (2000). The "what" and "why" of goal pursuits: Human needs and the self-determination of behaviour. Psychological Inquiry, 11, 227-268. doi: 10.1207/S15327965PLI1104_01

14. Deci, E. L., y Ryan, R. M. (2008). Facilitating optimal motivation and psychological well being across life's domain. Canadian Psychology, 49, 14-23.

15. Deci, E. L., y Ryan, R. M. (2012). Self-determination theory. En A. W. Kruglanski, P. A. M. Van Lange y E. T. Higgins (Eds.), Handbook of theories social psychology (Vol. 1, pp. 416-437). London: SAGE.

16. Faulkner, G., y Biddle, S. (2002). Mental health nursing and the promotion os physical activity. Journal Ment Health Nurs, 9(6), 659-665. doi: 10.1046/j.1365-2850.2002.00520.x

17. Fernández, R., y Manrique-Abril, F. G. (2010). Rol de la enfermería en el apoyo social del adulto mayor. Enfermería Global, 19, 1-9. doi: 10.4067/S0717-95532010000200009

18. Ferrand, C., Nasarre, S., Hautier, C., y Bonnefoy, M. (2012). Aging and well-being in french older adults regularly practicing physical activity: a self-determination perspective. Journal of Aging and Physical Activity, 20(2), 215-230. doi: 10.1007/s12603-011-0352-6

19. Graupera, J. L., Martínez del Castillo, J., y Martín, B. (2003). Factores motivacionales, actitudes y hábitos de práctica de actividad física en las mujeres mayores. Serie ICD de Investigación en Ciencias del Deporte, 35, 181-222.

20. González-Cutre, D., Sicilia, A., y Fernández, A. (2010). Hacia una mayor comprensión de la motivación en el ejercicio físico: medición de la regulación integrada en el contexto espańol. Psicothema, 22(4), 841-847.

21. Hair, J. F., Anderson, R. E., Tatham, R. L., y Black, W. C. (1998). Multivariate data analysis. Upper Saddle River, NJ: Prentice-Hall.

22. Hamer M., Batty, G. D., Kivimaki, M., y Stamatakis, E. (2011). Physical functional health and risk of future cardiovascular disease: the Scottish Health Survey. Archive of Internal Medicine, 171, 593-594. doi:10.1001/archinternmed.2011.86.

23. Hardcastle, S., y Taylor, A. H. (2001). Looking for more than weight loss and fitness gain: psychosocial dimensions among older women in a primary-care exercise-referral program. Journal Aging and physical activity, 9(3), 313-328.

24. Hellín, P., Moreno, J. A., y Rodríguez, P. L. (2004). Motivos de práctica físico-deportiva en la Región de Murcia. Cuadernos de Psicología del Deporte, 4(1-2), 101-116.

25. Krech, R. (2011). Social determinants of health: practical solutions to deal with a well-recognized issue. Bull World Health Organ, 89(10), 703. doi:10.2471/BLT.11.094870.

26. Marcos, P. J. (2010). Motivación en el ejercicio físico acuático: relación con la valoración, autonomía y disfrute del practicante. Tesis doctoral, Universidad Miguel Hernández de Elche.

27. Marcos, P. J., Borges, F., Rodríguez, A., Huéscar, E., y Moreno-Murcia, J. A. (2011). Indicios de cambio en los motivos de práctica físico-deportiva según el sexo y la edad. Apuntes de Psicología, 29, 123-132.

28. Martínez del Castillo, J., García, S., Graupera, J. L., Jiménez-Beatty, J. E., Martín, M., y Pouso, J. F. (2002). Hábitos y actitudes de las mujeres mayores en programas de actividad física en sociedades urbanas. Habilidad Motriz, 20, 5-12.

29. Moreno-Murcia, J. A., y Marcos, P. J. (2010). Estrategias motivacionales para programas de ejercicio físico acuático. Sevilla: Wanceulen.

30. Moreno, J. A., Cervelló, E., y Martínez, A. (2007). Validación de la Escala de Medida de los Motivos para la Actividad Física Revisada en español: Diferencias por motivos de participación. Anales de Psicología, 23(1), 167-176.

31. Moreno-Murcia, J. A., Cervelló, E., Borges, F., y Conte, L. (2009). El interés en la opinión del practicante de ejercicio físico tan importante papel en la predicción de la aptitud / de salud razón. Fitness and Performance Journal, 8(4), 247-253.

32. Moreno-Murcia, J. A., Águila, C., y Borges, F. (2011). La socialización en la práctica físico-deportiva de carácter recreativo: predictores de los motivos sociales. Apunts. Educación Física y Deportes, 103(1), 76-82.

33. Moreno, J. A., Cervelló, E., González-Cutre, D., Julián, J. A., y Del Villar, F. (2011). La motivación en el deporte. Claves para el éxito. Barcelona: Inde.

34. Moreno-Murcia, J. A., Huéscar, E., y Hellín, M. (2014). Mujer y deporte. Estrategias para su motivación. Barcelona: Inde.

35. Ostir, G. V., Cohen-Mansfield, J., Levielle, S. G., Volpato, S., y Guralnik, J. M. (2003). The association of positive and negative affect and 
exercise self-efficacy in older adults. Journal of Aging and Physical Activity, 11(2), 265-274.

36. Piedras-Jorge, C., Meléndez-Moral, J. C., y Tomás-Miguel, J. M. (2010). Beneficios del ejercicio físico en población mayor institucionalizada. Revista Española de Gerontologia, 45(3), 131-135. doi: 10.1016/j. regg.2009.10.012

37. Piéron, M. (1998). Perspectivas de la Educación Física en el siglo XXI. En J. Hernández, U. Castro, y H. Cruz. (Eds.), Educación física escolar y deporte de alto rendimiento (pp. 19-45). Las Palmas: ACCAFIDE.

38. Roth, D. L., Bachtler, S. D., y Fillingim, R. B. (1990). Acute emotional and cardiovascular effects of stressful mental work during aerobic exercise. Psychophysiology, 27, 694-701. doi: 10.1111/j.1469-8986.1990. tb03196.x

39. Ryan, R. M., Hicks, L., y Midgley, C. (1997). Social goals, academic goals, and avoiding seeking help in the classroom. Journal of Early Adolescence, 17, 152-171. doi:10.1177/0272431697017002003

40. Ryan, R. M., y Deci, E. L. (2000). Self-determination theory and the facilitation on intrinsic motivation, social development, and well-being. American Psychologist, 55, 68-78. doi: 10.1037 /0003-066X.55.1.68

41. Ryan, R. M., Frederick, C. M., Lepes, D., Rubio, N., y Sheldom, K. M. (1997). Intrinsic motivation and exercise adherence. International Journal of Sport Psychology, 28, 335-354.

42. Sánchez, J. M., y Núńez, J. L. (2007). Análisis preliminar de las propie- dades psicométricas de la versión espańola de la Escala de Necesidades Psicológicas Básicas en el Ejercicio Físico. Revista Iberoamericana de Psicología del Ejercicio y el Deporte, 2(2), 83-92.

43. Standage, M., y Treasure, D. C. (2002). Relationship among achievement goal orientations and multidimensional situational motivation in physical education. British Journal of Educational Psychology, 72(1), 87103. doi: $10.1348 / 000709902158784$

44. Vallerand, R. J. (1997). Toward a hierarchical model of intrinsic and extrinsic motivation. En M. Zanna (Ed.), Advances in experimental social psychology (pp. 271-360). New York: Academic Press.

45. Van Norman, K. (1998). Motivation and compliance in exercise programs for older adults. Journal of Physical Educatión, Recreation and Dance, 69(8), 24-27.

46. Vlachopoulos, S. P., y Michailidou, S. (2006). Development and initial validation of a measure of autonomy, competence, and relatedness: The Basic Psychological Needs in Exercise Scale. Measurement in Physical Education and Exercise Science, 10, 179-201. doi: 10.1207/s15327841mpee1003_4

47. Zimmer, Z., Martin, L. G., Jones, B. L. y Nagin, D. S. (2014). Examining late-life functional limitation trajectories and their associations with underlying onset, recovery, and mortality. Journals of Gerontology, Series B: Psychological Sciences and Social Sciences, 69, 275-286. doi:10.1093/geronb/gbt099. 